\title{
Identification of a novel fully human anti-toxic shock syndrome toxin (TSST)-1 single-chain fragment variable antibody averting TSST-1-induced mitogenesis and cytokines secretion
}

\section{Mahdieh Soezi \\ Pasteur Institute of Iran}

\section{Somayeh Piri-Gavgani}

Pasteur Institute of Iran

Behnoush Soltanmohammadi

Pasteur Institute of Iran

Mostafa Ghanei

Baqiyatallah University of Medical Sciences

\section{Farzam Vaziri}

Pasteur Institute of Iran

\section{Seyed Davar Siadat}

Pasteur Institute of Iran

\section{Abolfazl Fateh}

Pasteur Institute of Iran

\section{Shohreh Khatami}

Pasteur Institute of Iran

Fatemeh Rahimi-Jamnani ( $\nabla$ redogone.rahimii@gmail.com )

Pasteur Institute of Iran

\section{Research Article}

Keywords: Staphylococcus aureus, superantigens, toxic shock syndrome toxin-1, autoimmune diseases, toxic shock syndrome, fully human antibody, single-chain fragment variable

Posted Date: February 16th, 2022

DOI: https://doi.org/10.21203/rs.3.rs-1341632/v1

License: (9) This work is licensed under a Creative Commons Attribution 4.0 International License.

Read Full License 


\section{Abstract}

Background: Staphylococcal superantigens are virulence factors that help the pathogen escape the immune system and develop an infection. Toxic shock syndrome toxin (TSST)-1 is one of the most studied superantigens whose role in toxic shock syndrome and some particular disorders have been demonstrated. Inhibiting TSST-1 production with antibiotics and targeting TSST-1 with monoclonal antibodies might be one of the best strategies to prevent TSST-1-induced cytokines storm followed by lethality.

Results: A novel single-chain fragment variable (scFv), MS473, against TSST-1 was identified by selecting an scFv phage library on the TSST-1 protein. The MS473 scFv showed picomolar affinity and specific binding to TSST-1. Moreover, MS473 could significantly prevent TSST-1-induced mitogenicity and cytokine production.

Conclusions: Using traditional antibiotics with an anti-TSST-1 ScFv as a safe and effective agent leads to deleting the source of infection and prevents the detrimental effects of the toxin disseminated into the whole body.

\section{Background}

Staphylococcus aureus is one of the major causes of nosocomial and community-acquired infections, including skin and soft tissue infections, endocarditis, pneumonia, bacteremia, and toxic shock syndrome, the challenges facing the healthcare system in the world [1-3]. S. aureus is equipped with virulence factors with various functions, some of which have redundancy [1, 4]. Staphylococcal virulence factors range from cell wall-associated proteins to cytolysins and superantigens [3]. The staphylococcal superantigens such as staphylococcal enterotoxin serotype A (SEA), SEB, SEC, and toxic shock syndrome toxin (TSST)-1, interrupt the immune response against the pathogen through the antigen-independent activation of T cells and antigen-presenting cells (APCs) [5]. The concurrent binding of TSST- 1 to the $V$ beta domain of the T cell receptor (TCR) on T cells and the a-chain of major histocompatibility complex class II (MHC-II) on APCs begins a cascade of signaling pathways, leading to APCs and T cells hyperactivation and enormous cytokine secretion [5-7]. The nonspecific and hyperactivation of $T$ cells by TSST-1 can be the cause of some autoimmune diseases such as rheumatoid arthritis and psoriasis or emerge as acute infections and multi organs failure such as toxic shock syndrome [5, 8-10]. Functional strategies employed to inhibit TSST-1 include using antibiotics such as vancomycin, killing the pathogen, clindamycin or linezolid, preventing the protein synthesis, and direct targeting TSST-1 [3, 8, 11-14]. Given the emergence of resistant $S$. aureus strains and antibiotic-related side effects [1], specific targeting TSST1, disseminating to the organs [15], in combination with antibiotics, can lead to better results in patients. Monoclonal antibodies (mAbs) are the most functional agents used for toxin neutralization [16]. Of note, three mAbs, including bezlotoxumab against the toxin B of Clostridium difficile and raxibacumab and obiltoxaximab against the anthrax toxin of Bacillus anthracis, have been approved by the Food and Drug Administration of United States (FDA) $[1,16]$. In this regard, several studies reported mAbs against TSST- 
1, neutralizing the superantigenic effect of TSST-1 on human peripheral blood mononuclear cells (PBMCs) or murine spleen cells $[12,13,17]$, and protecting against TSST-1-induced lethality in animal models $[12,13]$. In contrast to the whole mAbs, great binding ability, high tissue penetration, and suitable clearance of antibody fragments such as single-chain fragment-variables (scFvs) make them superior neutralizing candidates $[8,18]$. The scFv comprises a heavy-chain variable domain $(\mathrm{VH})$ connected to a light-chain variable domain (VL) of an antibody by a peptide linker $[1,18]$. In this respect, Rukkawattanakul et al. generated three human anti-TSST-1 scFv antibodies, including HuscFv35, HuscFv53, and HuscFv56, inhibiting massive T cell proliferation and proinflammatory cytokine production promoted by TSST-1 [8].

In the current study, a fully human ScFv phage library was screened against the TSST-1 protein, leading to the identification of a novel ScFv with high affinity and specific binding to TSST-1. Next, we demonstrated the inhibition ability of the ScFv on the TSST-1-induced mitogenesis and cytokine release in vitro.

\section{Results}

\section{Selection of scFvs specific to TSST-1}

A fully human scFv phage display library was enriched against the recombinant TSST-1 protein, leading to the isolation of a pool of phages assessed based on their binding ability to the TSST-1 protein by the polyclonal-phage enzyme-linked immunosorbent assay (ELISA). As illustrated in Fig. 1A, the phages eluted from the third and fourth round of biopanning (output phages) on the TSST-1 protein had the highest signal intensities compared to other output phages and the controls. More than 500 Escherichia coli TG1 colonies infected with output phages of the third and fourth rounds of biopanning were evaluated for binding to TSST- 1 by the monoclonal-phage ELISA. Based on the results, 20 phage clones showed potential binding to the TSST-1 protein, among which five clones, MS457, MS460, MS465, MS473, and MS475, with the highest binding signal, were selected for further assessment (Fig. 1B) To generate soluble scFvs, $E$. col/HB 2151 bacteria were infected with phages of positive clones, including MS457, MS460, MS465, MS473, and MS475. Next, the expression of MS457, MS460, MS465, MS473, and MS475 was induced with isopropyl $\beta$-d-1-thiogalactopyranoside (IPTG), examined by sodium dodecyl sulphate polyacrylamide gel electrophoresis (SDS-PAGE). As shown in Fig. 2A, the expression yield of five scFvs ranged from 1 to $1.5 \mathrm{mg} / \mathrm{ml}$. Western blot assay demonstrated a single band at approximately 27 $\mathrm{kDa}$, corresponding to the scFv (Fig. 2B). Sequences analysis of five scFvs showed that MS457, MS473, MS475, MS460, and MS465 shared a common sequence. Therefore, MS473, having slightly higher expression levels $(\sim 1.5 \mathrm{mg} / \mathrm{ml})$, was selected for more analysis in the IMGT/V-QUEST database. Based on the results, the $\mathrm{V}$-regions of $\mathrm{VH}$ and $\mathrm{VL}$ domains of MS473 was derived from the human germline IGHV1-46*01 F (or IGHV1-46*03 F) and IGKV1-39*01 F (or IGKV1D-39*01 F), respectively.Furthermore, the $\mathrm{VH}$ and VL domains of MS473 had a complementarity determining region 3 (CDR3) length of 14 and 9 amino acids, respectively (Supplementary Fig. S2). 
The MS473 scFv was purified from the periplasmic extract using nicke/-nitrilotriacetic acid (Ni-NTA) resin, followed by dialysis. The concentration of purified and dialyzed scFv was about $400 \mu \mathrm{g} / \mathrm{ml}$. The results of SDS-PAGE showed a single band at approximately $27 \mathrm{kDa}$, corresponding to the expected size of the ScFv (Fig. 3A). The TSST-1 binding of MS473 was examined by ELISA. As illustrated in Fig. 3B, similar to mouse anti-staphylococcal TSST-1 mAb (Santa Cruz), MS473 showed strong TSST-1 binding compared to the controls.

\section{Reactivity of MS473 to TSST-1}

To determine the binding affinity ofMS473 to TSST-1, the TSST- 1 protein at concentrations of 1 and 2 $\mu \mathrm{g} / \mathrm{ml}$ was incubated with different concentrations of the scFv. The affinity constant $\left(K_{a f f}\right)$ was calculated using the formula described previously by Beatty et al. [19]. Based on the results, the $\mathrm{K}_{\text {aff }}$ of MS473 was $0.4 \mathrm{pM}^{-1}$.

The specific binding of MS473 to TSST-1 was appraised by ELISA using a group of proteins, including adiponectin, a-hemolysin, bovine serum albumin (BSA), and skimmed milk powder in addition to TSST-1. As shown in Fig. 4, MS473 had substantial binding to TSST-1 but not to other proteins tested.

\section{Inhibition of TSST-1-induced mitogenesis and cytokine secretion by MS473}

Human PBMCs were incubated with varying concentrations of TSST-1 $(1,10,25,50$, and $100 \mathrm{ng} / \mathrm{ml})$, and the results exhibited an equal mitogenic activity of TSST-1 at concentrations more than $50 \mathrm{ng} / \mathrm{ml}$ (Fig. 5A). Next, human PBMCs were treated concurrently with TSST-1 $(50 \mathrm{ng} / \mathrm{ml})$ and MS473 $(80 \mu \mathrm{g} / \mathrm{ml})$ to investigate the inhibition effect of MS473 on PBMCs proliferation induced by TSST-1. As shown in Fig. 5B, MS473 could significantly inhibit the proliferative effect of TSST-1 and decrease the number of lymphocyte colonies in the wells containing human PBMCs incubated with TSST-1 and MS473 in comparison with a large number of clumps observed in the control group (the cells treated with TSST-1 and phosphate buffer saline [PBS]). Furthermore, treatment of human PBMCs with TSST-1 and MS473 led to the decreased secretion of interleukin (IL)-2, IL-4, IL-5, IL-6, IL-10, IL-12, IL-13, IL-17A, interferon (IFN)$\gamma$, tumor necrosis factor (TNF)-a, granulocyte colony-stimulating factor (G-CSF), and transforming growth factor (TGF)- $\beta$ compared to the control group (human PBMCs treated with TSST-1) (Fig. 6).

\section{Discussion}

The possible involvement of TSST-1 in a group of complicated disorders, from atherosclerosis [20] and autoimmune diseases (e.g. rheumatoid arthritis, psoriasis) [3, 9, 21], to Kawasaki syndrome and sudden infant death syndrome $[9,21]$, plus its prominent role in the toxic shock syndrome [5, 22], highlight the need to find functional agents specifically targeting TSST-1. The history of applying antibodies as neutralizing toxins and three FDA-approved mAbs in the market (bezlotoxumab, obiltoxaximab, and Raxibacumab) [16] make mAbs the first choice as anti-TSST-1 agents. Although few mAbs targeting TSST-1 have been developed [12,13], scFv antibodies have particular characteristics, such as small size, high tissue penetration, low immunogenicity, and low-cost and easy production, making them functional 
toxin-neutralizing agents compared to conventional antibodies $[1,8,18]$. To isolate TSST-1-specific scFvs, we enriched a fully human ScFv phage library was against the TSST-1 protein in this study. One novel scFv, MS473, was identified, and its binding reactivity was assessed. The MS473 scFv showed highaffinity binding to TSST-1 $\left(\mathrm{K}_{a f f}=0.4 \mathrm{pM}^{-1}\right)$. The specific binding of MS473 to TSST-1 compared to the control proteins, including adiponectin, a-hemolysin, BSA, and skimmed milk powder, was appraised by ELISA, and the results demonstrated the specific binding of MS473 to the target toxin (TSST-1).

TSST-1 exerts its mitogenic activity by bridging TCRs on T cells and MHC II on APCs in an antigenindependent manner [5]. Of note, Huseby et al. showed that the treatment of the mononuclear cells with TSST-1 $(1 \mu \mathrm{g} /$ well) resulted in the formation of lymphocytes colonies as brown clumps [23]. In this respect, we treated human PBMCs with TSST-1 $(50 \mathrm{ng} / \mathrm{ml})$ and MS473 $(80 \mu \mathrm{g} / \mathrm{ml})$ to determine whether the binding of the SCFv to TSST-1 led to the inhibition of the proliferation of PBMCs stimulated with TSST1. Based on the results, MS473 had a significant inhibition effect on PBMCs proliferation and reduced the number of colony-forming cells. The effect of MS473 on the secretion of a group of cytokines from PBMCs induced by TSST-1 was also evaluated by a Multi-Analyte ELISArray Kit. The data indicated that MS473 could decrease the release of cytokines, including IL-2, IL-4, IL-5, IL-6, IL-10, IL-12, IL-13, IL-17A, IFN- - , TNF- $a$, G-CSF, and TGF- $\beta$ from TSST-1-stimulated PBMCs in comparison with PBMCs treated with TSST-1 alone. The human PBMC population consists of various cells, including B cells, dendritic cells, monocytes, natural killer cells, $C D 8^{+} T$ cytotoxic cells, and $C D 4^{+} T$ helper (h) cells (e.g. naïve $T$ cells, Th1, Th2, Th17, and T regulatory) $[24,25]$. Therefore, TSST-1-mediated activation of APCs and CD $4^{+} \mathrm{T}$ cell subsets in PBMCs might lead to the secretion of a cocktail of cytokines ranging from proinflammatory to inhibitory cytokines [5, 21, 26-28]. In contrast, the neutralization of TSST- 1 by MS473 could prevent TSST1-induced cytokines production in PBMCs. In this regard, Bonventre et al. developed a murine $\lg \mathrm{G} \mathrm{mAb}$ against TSST-1, designated MAb 8-5-7, blocking TSST-1-induced mitogenesis in murine spleen cells, inhibiting IL-1 production from human PBMCs stimulated with TSST-1, and protecting the rabbits against TSST-1-induced lethality [12]. In another study, Kum et al. reported that MAb5, a mouse IgG1 mAb, had a significant inhibitory effect on TSST-1-induced mitogenicity, and IL-1 $\beta$, IL-6, and TNF-a secretion in human PBMCs [13]. Moreover, they showed that MAb5 prolonged the survival rate in the subcutaneous TSST-1 infusion rabbit model and the D-galactosamine sensitized murine model of lethal shock [13]. In the study by Pang et al., MAb5 had a neutralization effect on SEB-induced superantigenic activity in human PBMCs (e.g. inhibition of SEB-induced mitogenicity and TNF-a release) [29]. However, its neutralization activity was 1000-fold lower than against TSST-1 [29]. Similarly, Kum and Chow showed that although MAb5 inhibited SEA-induced mitogenicity and TNF-a production in human PBMCs, and protected mice against SEA-induced lethality, its inhibition potency against SEA was lower than against TSST-1, resulting from the lower binding affinity of MAb5 to SEA $\left(780 \mathrm{nM}^{-1}\right)$ compared to TSST-1 $\left(0.9 \mathrm{nM}^{-1}\right)$ [30]. Consistent with our study, Rukkawattanakul et al. isolated three anti-TSST-1 scFv antibodies (HuscFv35, HuscFv53, and HuscFv56) from a human scFv phage library [8]. To assess the neutralization activity of HuscFv35, HuscFv53, and HuscFv56, human PBMCs (5 $10^{4}$ cells/well) were incubated with the TSST-1 protein $(1000 \mathrm{ng} / \mathrm{ml})$ and the HuscFv $(4 \mu \mathrm{g} /$ well) for 24 hours. Based on the results, HuscFv35 and HuscFv56 exhibited more inhibitory effects on the proliferation of T cells and production of IL-1 $\beta$, IL- 6 , and TNF- $a$ in 
PBMCs induced by TSST-1 than that of HuscFv53 [8]. Taken together, MS473 displayed high-affinity binding to TSST-1 (with the $\mathrm{K}_{a f f}$ in the picomolar range), leading to the inhibition of PBMCs proliferation and a significant reduction in the secretion of a group of inflammatory and anti-inflammatory cytokines, including IL-2, IL-4, IL-5, IL-6, IL-10, IL-12, IL-13, IL-17A, IFN- $\gamma$, TNF-a, G-CSF, and TGF- $\beta$.

\section{Conclusions}

We developed a high affinity fully human ScFv, MS473, with specific binding ability to TSST-1. The MS473 scFv could significantly affect TSST-1-induced mitogenesis and decrease the release of an array of cytokines from human PBMCs stimulated with TSST-1. Moreover, the protective activity of MS473 against TSST-1-induced lethality is also being evaluated in a D-galactosamine-sensitized mouse model of lethal shock. Given the role of TSST-1 in toxic shock syndrome and some disorders with unknown causes, the use of conventional antibiotics in combination with a neutralizing scFv with substantial pharmacokinetic and pharmacodynamic profiles might lead to encouraging results in patients with complicated conditions.

\section{Methods}

\section{Isolation of phages expressing scFvs specific to TSST-1}

To isolate TSST-1-specific phages, a fully human scFv phage library with total diversity of $2 \times 10^{10}$ was enriched against the TSST-1 protein as described previously with some modifications [1, 31]. Briefly, a 96well MaxiSorp plate (Nunc, Roskilde, Denmark) was coated with $100 \mu$ l of the TSST-1 protein $(2 \mu \mathrm{g} / \mathrm{ml}$ in $\mathrm{NaHCO3}$ ) (Sigma-Aldrich, St. Louis, MO, USA) overnight at $4^{\circ} \mathrm{C}$. Following blocking the plate $(200 \mu \mathrm{l} ; 15$ $\mathrm{mg} / \mathrm{ml}$ BSA in PBS containing 0.1\% tween-20 [PBS-T]), pre-blocked phages ( $10^{12}$ plaque-forming unit $/ \mathrm{ml}$ ) amplified from the scFv phage library were added to the wells, and incubation was done for 90 minutes at room temperature (RT). Following several times washing with PBS-T, bound phages were eluted (output) and amplified (input) for further biopanning rounds. This procedure was repeated for four rounds. Washing steps were increased from round one (10 times) to round four ( 25 times). The output/input ratio of each round was determined to evaluate enrichment efficiency.

The polyclonal-phage ELISA was carried out to determine which biopanning round contained the phages specific to TSST-1 [31]. In brief, a 96-well MaxiSorp plate was coated with $100 \mu$ l of the TSST-1 protein (2 $\mu \mathrm{g} / \mathrm{ml}$ ) or BSA $(2 \mu \mathrm{g} / \mathrm{ml})$ (Merck, Darmstadt, Germany) overnight at $4^{\circ} \mathrm{C}$. The wells were blocked, followed by the incubation with output phages obtained from the first to the fourth round of biopanning (output $1^{-}$ output $_{4}$ ) for 60 minutes at RT. After multiple washing steps with PBS-T, horseradish peroxidase (HRP)conjugated mouse anti-M13 antibody (1:2000 dilution in blocking buffer) (Santa Cruz Biotechnology, INC.) was added to the wells, followed by the incubation for 60 minutes at RT. Next, the wells were washed several times with PBS-T, and the signals were generated by adding 3,3',5,5'-

Tetramethylbenzidine (TMB) (Thermo Scientific, MA, US). The reactions were stopped with sulfuric acid 
(1 M) (Merck), and the absorbance at $450 \mathrm{~nm}$ was measured using a microplate reader (Epoch, BioTek, USA).

Based on the data obtained from the polyclonal-phage ELISA, output phages of the third and fourth rounds of biopanning (output ${ }_{3}$ and output ${ }_{4}$ ), showing the highest signal intensity compared to the control, were further assessed by the monoclonal-phage ELISA [31]. Briefly, E. coli TG1 bacteria were infected with output phages (output ${ }_{3}$ and output ${ }_{4}$ ) and cultured on lysogeny broth (LB) agar (Merck) plates with $150 \mu \mathrm{g} / \mathrm{ml}$ ampicillin (Sigma-Aldrich). After incubation overnight at $37^{\circ} \mathrm{C}$, the colonies were picked up randomly, and phage amplification was done as described previously [1]. The binding ability of phages to the TSST-1 protein was investigated by ELISA, as mentioned above in the polyclonal-phage ELISA.

\section{Expression}

Five phage clones, MS457, MS460, MS465, MS473, and MS475, which showed the highest binding to the TSST-1 protein compared to the control in the monoclonal-phage ELISA, were selected for more evaluations. To produce soluble ScFv antibodies, the non-suppressor $E$. coli strain, $\mathrm{HB}_{2151}$, was infected with the phages obtained from five phage clones and cultured on LB agar plates containing ampicillin, followed by the incubation overnight at $37^{\circ} \mathrm{C}[1,32]$. The single colonies were picked up and cultured in terrific broth containing $100 \mu \mathrm{g} / \mathrm{ml}$ ampicillin. After adding IPTG $(0.1 \mathrm{mM})$ (Thermo Scientific) and incubating overnight at $24^{\circ} \mathrm{C}$, the cultures were centrifuged, and the pellets were incubated with the lysis buffer for 60 minutes at RT [32,33]. Next, the existing level of scFvs in the periplasmic fraction of $E$. coli $\mathrm{HB}_{2151}$ bacteria, carrying the phagemids (MS457, MS460, MS465, MS473, or MS475), was investigated by a $12 \%$ SDS-PAGE gel, followed by western blot analysis. After electrophoresis, the proteins were transferred from the SDS-PAGE gel (12\%) onto polyvinylidene fluoride (PVDF) membrane (GE Healthcare, Little Chalfont, UK). The blocked membrane was incubated with mouse anti-human scFv polyclonal antibody (1:200 dilution) for 60 minutes at RT [1]. After several washing steps with tris-buffered saline (TBS) with $0.05 \%$ tween-20 (TBS-T) and TBS, the membrane was incubated with goat anti-mouse IgGHRP-conjugated antibody (1:2000 dilution) (Santa Cruz) for 60 minutes at RT, followed by several washing steps and addition of 3,3'-diaminobenzidine substrate (DAB) (Sigma-Aldrich) and hydrogen peroxide $\left(\mathrm{H}_{2} \mathrm{O}_{2}\right)$ (Merck).

\section{Sequencing}

The phagemid DNA of clones MS457, MS460, MS465, MS473, and MS475, was purified using the High Pure Plasmid Isolation Kit (Roche, Mannheim, Germany), based on the manufacturer's recommendation. For sequencing, the forward primer, 5'- CTA TGA CCA TGA TTA CGA ATT TCT A -3', was used. The nucleotide sequences of five scFvs were appraised using the Gene Runner program (version 6.0). Furthermore, the amino acid sequences of V-regions of MS473 was analyzed by the IMGT/V-QUEST tool (http://www.imgt.org/IMGT_vquest/analysis) [1]. 


\section{Assessment of the binding ability of the purified ScFv to TSST-1}

The soluble scFv, MS473, was purified using a Ni-NTA column (Qiagen, Hilden, Germany), according to the manufacturer's instructions. The bound proteins were eluted with $200 \mathrm{mM}$ imidazole (Merck). Next, all the eluted fractions were pooled and then placed in a dialysis bag (cut off $14 \mathrm{kDa}$, Sigma-Aldrich), according to the manufacturer's instructions. The concentration of purified and dialyzed scFv (MS473) was measured via the Bradford assay. Moreover, the purity of the scFv was analyzed by a $12 \%$ SDS-PAGE gel.

The binding ability of purified SCFv to TSST-1 was determined by ELISA as described previously with some modifications [31]. Briefly, a 96-well MaxiSorp plate was coated with $100 \mu$ l of the TSST-1 protein (2 $\mu \mathrm{g} / \mathrm{ml})$ or BSA $(2 \mu \mathrm{g} / \mathrm{ml})$ (as the control). Next, the wells were blocked and then incubated with MS473 or SP220 (an scFv against staphylococcal a-hemolysin) $(400 \mu \mathrm{g} / \mathrm{ml})$ for 60 minutes at RT. After several washing steps, mouse anti-human scFv polyclonal antibody was added to the wells, and incubation was done for 60 minutes at RT. The wells were washed several times with PBS-T and PBS, and goat antimouse IgG-HRP-conjugated antibody was added to the wells, followed by incubation for 60 minutes at RT. Moreover, the TSST-1-coated wells incubated with mouse anti-staphylococcal TSST-1 mAb (1/1000 dilution) (Santa Cruz), followed by goat anti-mouse IgG-HRP-conjugated antibody were used as the positive control. After multiple washing steps, the TMB substrate solution was added to the wells, and the color reactions were stopped with sulfuric acid. A microplate reader determined the absorbance at 450 $\mathrm{nm}$.

\section{Affinity determination}

The binding affinity of MS473 to TSST-1 was determined as described previously [19, 31, 34]. In brief, a 96-well MaxiSorp plate was coated with $100 \mu \mathrm{l}$ of the TSST-1 protein ( 1 and $2 \mu \mathrm{g} / \mathrm{ml})$. After blocking, the wells were incubated with serial dilutions of the MS473 scFv $(0.02-500 \mu \mathrm{g} / \mathrm{ml})$ for 60 minutes at RT. After several washing steps, mouse anti-human scFv polyclonal antibody was added to the wells, and incubation was done for 60 minutes at RT. Next, the wells were washed multiple times, followed by the incubation with goat anti-mouse IgG-HRP-conjugated antibody for 60 minutes at RT. After adding the TMB substrate solution, the color development was stopped with sulfuric acid. The absorbance was read at $450 \mathrm{~nm}$. The $\mathrm{K}_{\text {aff }}$ of MS473 to the TSST-1 protein was measured using the following formula:

$\mathrm{Ag} / \mathrm{Ag}^{\prime}=\mathrm{n}$

$\mathrm{K}_{a f f}=\mathrm{n}-1 / 2\left(\mathrm{n}\left[\mathrm{scFv} \mathrm{v}^{\prime}\right]-[\mathrm{scFv}]\right)$

Where Ag and Ag' are the TSST-1 protein at concentrations of 2 and $1 \mu \mathrm{g} / \mathrm{ml}$, respectively; the scFv and scFv' are the concentration of MS473 at OD-50 and OD-50' for the wells coated with the TSST-1 protein at concentrations of 2 and $1 \mu \mathrm{g} / \mathrm{ml}$, respectively.

\section{Specificity}


The binding specificity of MS473 to TSST-1 was assayed by ELISA as described previously [31]. In brief, a 96-well MaxiSorp plate was coated with $100 \mu \mathrm{l}$ of the TSST-1 protein $(2 \mu \mathrm{g} / \mathrm{ml})$, the adiponectin protein (2 $\mu \mathrm{g} / \mathrm{ml}$ ) (R\&D Systems, Minnesota, US), the a-hemolysin protein $(2 \mu \mathrm{g} / \mathrm{ml}$ ) (Merck, Calbiochem, Germany), BSA $(2 \mu \mathrm{g} / \mathrm{ml})$, and skimmed milk powder $(1 \mathrm{mg} / \mathrm{ml})$. After blocking, the wells were individually incubated with MS473. The wells were washed several times, and mouse anti-human scFv polyclonal antibody was added to the wells, followed by goat anti-mouse IgG-HRP-conjugated antibody. Next, the TMB substrate solution was added. After stopping the reaction with sulfuric acid, the absorbance at $450 \mathrm{~nm}$ was read using a microplate reader.

\section{Assessment of the inhibition ability of MS473 on the TSST-1-induced mitogenesis and cytokine release in human PBMCs}

The neutralizing activity of MS473 against the superantigenic activity of TSST-1 was examined on human PBMCs isolated from the whole blood of two healthy donors (men, 50 and 45 years) as described previously with some modifications $[8,12,13,29,35,36]$. To determine the effective proliferative dose of TSST-1, fresh human PBMCs ( $10^{6} \mathrm{cells} / \mathrm{ml}$ ) in RPMI 1640 medium (Gibco; Grand Island, NY, USA) with L-glutamine (2 mM) (Gibco) supplemented with heat-inactivated fetal bovine serum (10\%) (Gibco) were seeded in 96-well round-bottom tissue culture plates (JET BIOFIL, Guangzhou, China) and were incubated with the TSST- 1 protein at concentrations of $1,10,25,50$, and $100 \mathrm{ng} / \mathrm{ml}$ for 24 hours at $37^{\circ} \mathrm{C}, 5 \% \mathrm{CO} 2$. The proliferation of PBMCs and the formation of cell clumps induced by TSST-1 were investigated using an inverted microscope [23]. Next, the cells were incubated concurrently with the TSST-1 protein (50 $\mathrm{ng} / \mathrm{ml})$ and MS473 $(80 \mu \mathrm{g} / \mathrm{ml})$ for 24 hours at $37^{\circ} \mathrm{C}, 5 \% \mathrm{CO} 2$. The cells incubated with PBS or the TSST-1 protein $(50 \mathrm{ng} / \mathrm{ml})$ and PBS were used as the controls. The inhibitory effect of MS473 on the proliferation of PBMCs stimulated with TSST-1 was assessed by an inverted microscope. Moreover, the culture supernatant of PBMCs treated simultaneously with the TSST-1 protein $(50 \mathrm{ng} / \mathrm{ml})$ and MS473 $(80 \mu \mathrm{g} / \mathrm{ml})$ were collected after 24 hours incubation at $37^{\circ} \mathrm{C}, 5 \% \mathrm{CO} 2$, followed by the centrifugation at $1000 \mathrm{~g}$ for 5 min. The cells treated with PBS or the TSST-1 protein $(50 \mathrm{ng} / \mathrm{ml})$ and PBS served as the controls. The concentrations of human IL-2, IL-4, IL-5, IL-6, IL-10, IL-12, IL-13, IL-17A, IFN- $\gamma$, TNF- $a$, G-CSF, and TGF- $\beta$ in the culture supernatant of PBMCs (stimulated and unstimulated) were calculated using the standard curve provided in a Multi-Analyte ELISArray Kit (Qiagen), according to the manufacturer's instructions. Experimental procedures with human blood were approved by the Ethics Committee of the Pasteur Institute of Iran and were done in accordance with the Helsinki Declaration. The participant provided written informed consent before enrollment.

\section{Statistical analyses}

The one-way analysis of variance (ANOVA) and Student's $t$ test were carried out in GraphPad Prism version v.6.0.7. The $P$-value $<0.05$ was considered significant.

\section{Declarations}


Acknowledgments

We thank the Mycobacteriology and Pulmonary Research Department of Pasteur Institute of Iran for their cooperation.

\section{Authors' contributions}

F.R.J. conceived and designed the study. M.G., S.D.S., S.K., and A.F. helped design the study. M.S. performed the experiments. M.S., S.P.G., and B.SM. were involved in the manuscript preparation. All authors read and approved the final manuscript.

\section{Funding}

This article was extracted from the Ph.D. thesis of Mahdieh Soezi, granted by the Pasteur Institute of Iran.

\section{Availability of data and materials}

All data generated or analyzed during this study are included in this manuscript and its Supplementary Information file.

\section{Ethics approval and consent to participate}

Experimental procedures with human blood were approved by the Ethics Committee of the Pasteur Institute of Iran and were done in accordance with the Helsinki Declaration. The participant provided written informed consent before enrollment.

\section{Consent for publication}

Not applicable.

\section{Competing interests}

The authors declare that they have no competing interests.

\section{References}

1. Soltanmohammadi B, Piri-Gavgani S, Basardeh E, Ghanei M, Azizi M, Khaksar Z, et al. Bactericidal fully human single-chain fragment variable antibodies protect mice against methicillin-resistant Staphylococcus aureus bacteraemia. Clin TransI Immunol. 2021;10:e1302. doi:10.1002/cti2.1302.

2. Larkin EA, Stiles BG, Ulrich RG. Inhibition of toxic shock by human monoclonal antibodies against staphylococcal enterotoxin B. PLoS ONE. 2010;5:e13253. doi:10.1371/journal.pone.0013253.

3. Lin Y-C, Peterson ML. New insights into the prevention of staphylococcal infections and toxic shock syndrome. Expert Rev Clin Pharmacol. 2010;3:753-7. 
4. Kong C, Chee CF, Richter K, Thomas N, Abd Rahman N, Nathan S. Suppression of Staphylococcus aureus biofilm formation and virulence by a benzimidazole derivative, UM-C162. Sci Rep. 2018;8:2758.

5. Hu D. L, Li S, Fang R, Ono HK. Update on molecular diversity and multipathogenicity of staphylococcal superantigen toxins. Anim Dis. 2021;1:1-15.

6. Schlievert PM, Davis CC. Device-associated menstrual toxic shock syndrome. Clin Microbiol Rev. 2020;33:e00032-00019. doi: 10.1128/CMR.00032-19.

7. Spaulding AR, Salgado-Pabón W, Kohler PL, Horswill AR, Leung DY, Schlievert PM. Staphylococcal and streptococcal superantigen exotoxins. Clin Microbiol Rev. 2013;26:422-7.

8. Rukkawattanakul T, Sookrung N, Seesuay W, Onlamoon N, Diraphat P, Chaicumpa W, et al. Human scFvs that counteract bioactivities of Staphylococcus aureus TSST-1. Toxins. 2017;9:50.

9. Dinges MM, Orwin PM, Schlievert PM. Exotoxins of Staphylococcus aureus. Clin Microbiol Rev. 2000;13:16-34.

10. Tong SY, Davis JS, Eichenberger E, Holland TL, Fowler VG. Staphylococcus aureus infections: epidemiology, pathophysiology, clinical manifestations, and management. Clin Microbiol Rev. 2015;28:603-61.

11. Hodille E, Rose W, Diep BA, Goutelle S, Lina G, Dumitrescu O. The Role of Antibiotics in Modulating Virulence in Staphylococcus aureus. Clin Microbiol Rev. 2017;30:887-917.

12. Bonventre PF, Thompson M, Adinolfi L, Gillis Z, Parsonnet J. Neutralization of toxic shock syndrome toxin-1 by monoclonal antibodies in vitro and in vivo. Infect Immun. 1988;56:135-41.

13. Kum WW, Laupland KB, Chow AW. Defining a novel domain of staphylococcal toxic shock syndrome toxin-1 critical for major histocompatibility complex class II binding, superantigenic activity, and lethality. Can J Microbiol. 2000;46:171-9.

14. Berger S, Kunerl A, Wasmuth S, Tierno P, Wagner K, Brügger J. Menstrual toxic shock syndrome: case report and systematic review of the literature. Lancet Infect Dis. 2019;19:313-21.

15. Bonventre PF, Heeg H, Cullen C, Lian CJ. Toxicity of recombinant toxic shock syndrome toxin 1 and mutant toxins produced by Staphylococcus aureus in a rabbit infection model of toxic shock syndrome. Infect Immun. 1993;61:793-9.

16. Wang-Lin SX, Balthasar JP: Pharmacokinetic and pharmacodynamic considerations for the use of monoclonal antibodies in the treatment of bacterial infections. Antibodies. 2018;7:5.

17. Verdrengh M, Kum W, Chow A, Tarkowski A. The effect of site-specific monoclonal antibodies directed to toxic shock syndrome toxin-1 in experimental Staphylococcus aureus arthritis. Clin Exp Immunol. 1999;118:268-70.

18. Ahamadi-Fesharaki R, Fateh A, Vaziri F, Solgi G, Siadat SD, Mahboudi F, et al. Single-chain variable fragment-based bispecific antibodies: Hitting two targets with one sophisticated arrow. Mol ther oncolytics. 2019;14:38. 
19. Beatty JD, Beatty BG, Vlahos WG. Measurement of monoclonal antibody affinity by non-competitive enzyme immunoassay. J Immunol Methods. 1987;100:173-9.

20. Zhao H, Chen L, He C, Li S, Yang H, Xu X, Hu F, Cui Z, Liu, et al. Chronic Staphylococcus aureus Superantigen Toxic Shock Syndrome Toxin-1 Exposure Accelerates the Progression of Atherosclerosis in Rabbits. Acta Cardiol Sin. 2020;36:24-32.

21. Yarwood JM, Leung DY, Schlievert PM. Evidence for the involvement of bacterial superantigens in psoriasis, atopic dermatitis, and Kawasaki syndrome. FEMS Microbiol Lett. 2000;192:1-7.

22. Kang D, Lin CH, Chen G, Guo SG, Wu YS, Zheng ZP, et al. Interaction of toxin-1 and T lymphocytes in toxic shock syndrome. Front Biosci Landmark Ed. 2014;19:571-7.

23. Huseby M, Shi K, Brown CK, Digre J, Mengistu F, Seo KS, , et al. Structure and biological activities of beta toxin from Staphylococcus aureus. J Bacteriol. 2007;189:8719-26.

24. Rajagopalan G, Tilahun AY, Asmann YW, David CS. Early gene expression changes induced by the bacterial superantigen staphylococcal enterotoxin $B$ and its modulation by a proteasome inhibitor. Physiol genom. 2009;37:279-93.

25. Kleiveland CR. Peripheral Blood Mononuclear Cells. In: Verhoeckx K, Cotter P, López-Expósito I, et al., editors. The Impact of Food Bioactives on Health: in vitro and ex vivo models [Internet]. Cham $(\mathrm{CH})$ : Springer; 2015. p. 161-7 doi: 10.1007/978-3-319-16104-4_15.

26. Fang P, Li X, Dai J, Cole L, Camacho JA, Zhang Y, et al. Immune cell subset differentiation and tissue inflammation. J Hematol Oncol. 2018;11:97.

27. Choy E, Rose-John S. Interleukin-6 as a multifunctional regulator: inflammation, immune response, and fibrosis. J. Scleroderma Relat Disord. 2017;2 Supp 2:1-5.

28. Oster W, Lindemann A, Mertelsmann R, Herrmann F: Production of macrophage-, granulocyte-, granulocyte-macrophage-and multi-colony-stimulating factor by peripheral blood cells. Eur $\mathrm{J}$ Immunol 1989; 19:543-8.

29. Pang LT, Kum WW, Chow AW. Inhibition of staphylococcal enterotoxin B-induced lymphocyte proliferation and tumor necrosis factor alpha secretion by MAb5, an anti-toxic shock syndrome toxin 1 monoclonal antibody. Infect Immun. 2000;68:3261-8.

30. Kum WW, Chow AW. Inhibition of staphylococcal enterotoxin A-induced superantigenic and lethal activities by a monoclonal antibody to toxic shock syndrome toxin-1. J Infec Dis. 2001;183:1739-48.

31. Jamnani FR, Rahbarizadeh F, Shokrgozar MA, Ahmadvand D, Mahboudi F, Sharifzadeh Z. Targeting high affinity and epitope-distinct oligoclonal nanobodies to HER2 over-expressing tumor cells. Exp Cell Res. 2012;318:1112-24.

32. Eisenhardt SU, Schwarz M, Bassler N, Peter K. Subtractive single-chain antibody (scFv) phagedisplay: tailoring phage-display for high specificity against function-specific conformations of cell membrane molecules. Nat Protoc. 2007;2:3063-73.

33. Rahumatullah A, Ahmad A, Noordin R, Lai JY, Baharudeen Z, Lim TS. Applicability of Brugia malayi immune antibody library for the isolation of a human recombinant monoclonal antibody to 
Echinococcus granulosus antigen B. Exp Parasitol. 2020;219:108029.

https://doi.org/10.1016/j.exppara.2020.108029.

34. Mazaheri S, Talebkhan Y, Mahboudi F, Nematollahi L, Cohan RA, Ardakani EM, et al. Improvement of Certolizumab Fab' properties by PASylation technology. Sci Rep. 2020;10:1-13.

35. Xiao J, Zhu F, Liu X, Xiong J. Th1/Th2/Th17/Treg expression in cultured PBMCs with antiphospholipid antibodies. Mol Med Rep. 2012;6:1035-9.

36. Gonzalez Rivas E, Ximenez C, Nieves-Ramirez ME, Moran Silva P, Partida-Rodríguez O, Hernandez EH, et al. Entamoeba histolytica calreticulin induces the expression of cytokines in peripheral blood mononuclear cells isolated from patients with amebic liver abscess. Front. Cell Infect Microbiol. 2018;8:358.

\section{Figures}

A

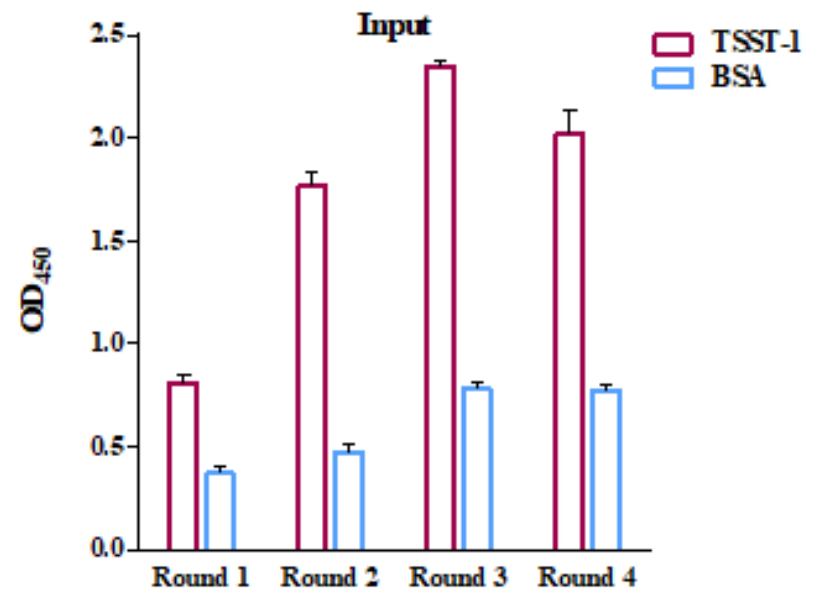

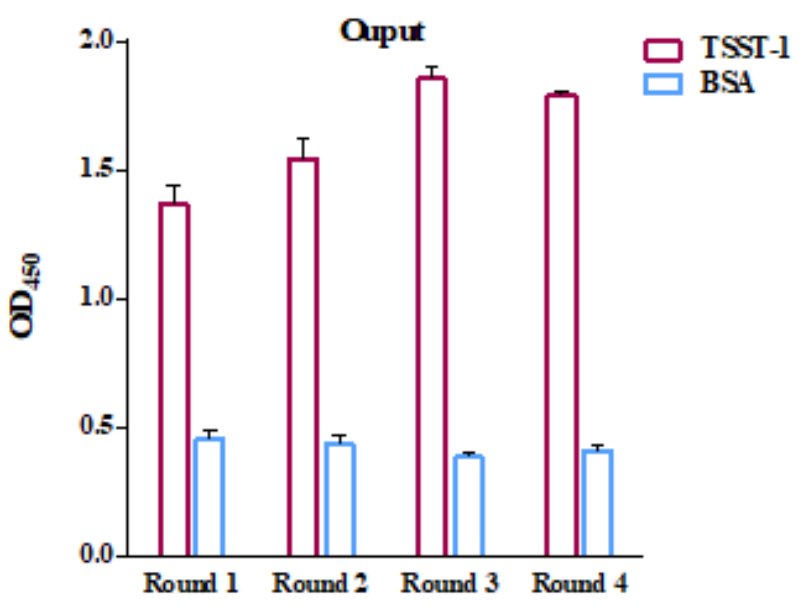

TSST-1

BSA

Figure 1 
The binding properties of purified phages to TSST-1. The TSST-1-binding of phages obtained from four rounds of biopanning on the TSST-1 protein was assessed by (A) the polyclonal-phage ELISA and (B) monoclonal-phage ELISA. (A) The wells coated with TSST-1 or bovine serum albumin (BSA) were incubated with the selected phages, followed by horseradish peroxidase (HRP)-conjugated mouse antiM13 antibody. The highest signal intensities were observed with output phages of the third and fourth round of biopanning on the TSST-1 protein compared to other output phages and the controls. (B) Twenty phage clones showed potential binding to the TSST-1 protein, among which five clones, MS457, MS460, MS465, MS473, and MS475, with the highest binding signal, were selected for further assessment. Samples were run in triplicate, and the results are expressed as mean \pm SEM of at least three individual experiments. Statistical comparisons were carried out with Student's $t$-test. ${ }^{*} P<0.05,{ }^{\star} P<0.01$.

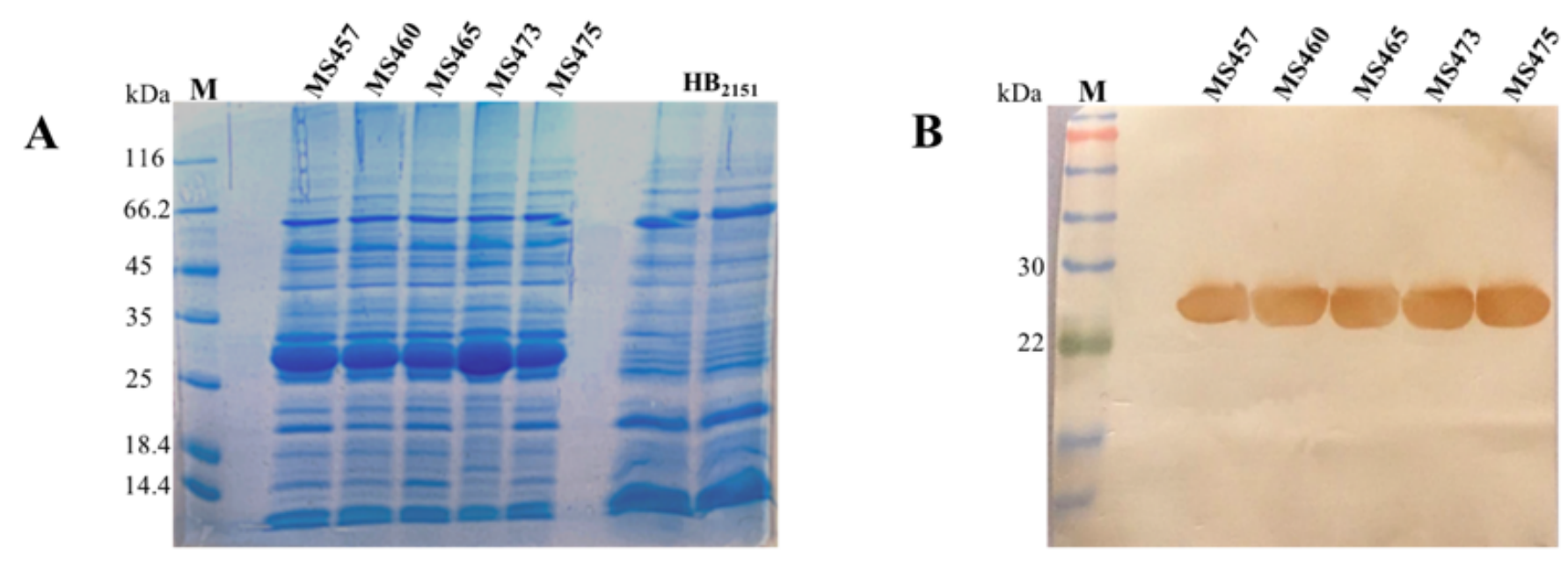

Figure 2

The expression of five soluble scFvs in E. coli $\mathrm{HB}_{2151}$. The expression of five scFvs, MS457, MS460, MS465, MS473, and MS475, in E. coli $\mathrm{HB}_{2151}$, was evaluated by (A) SDS-PAGE and (B) western blot analysis. (A) The expression level of MS457, MS460, MS465, MS473, and MS475 ( $27 \mathrm{kDa})$ in the periplasmic extract of E.coli $\mathrm{HB}_{2151}$ infected with the selected phagemids and the periplasmic extract of uninfected E.coli $\mathrm{HB}_{2151}$ (control) induced by $0.1 \mathrm{mM}$ IPTG were assessed by an SDS-PAGE gel (12\%). (B) A single band of the expected size $(\sim 27 \mathrm{kDa})$, related to the $\mathrm{scFv}$, was detected by probing with mouse anti-human scFv polyclonal antibody, followed by goat anti-mouse IgG-horseradish peroxidase (HRP)-conjugated antibody. Lane M: pre-stained protein marker. The original images can be found in Supplementary Fig. S1. 

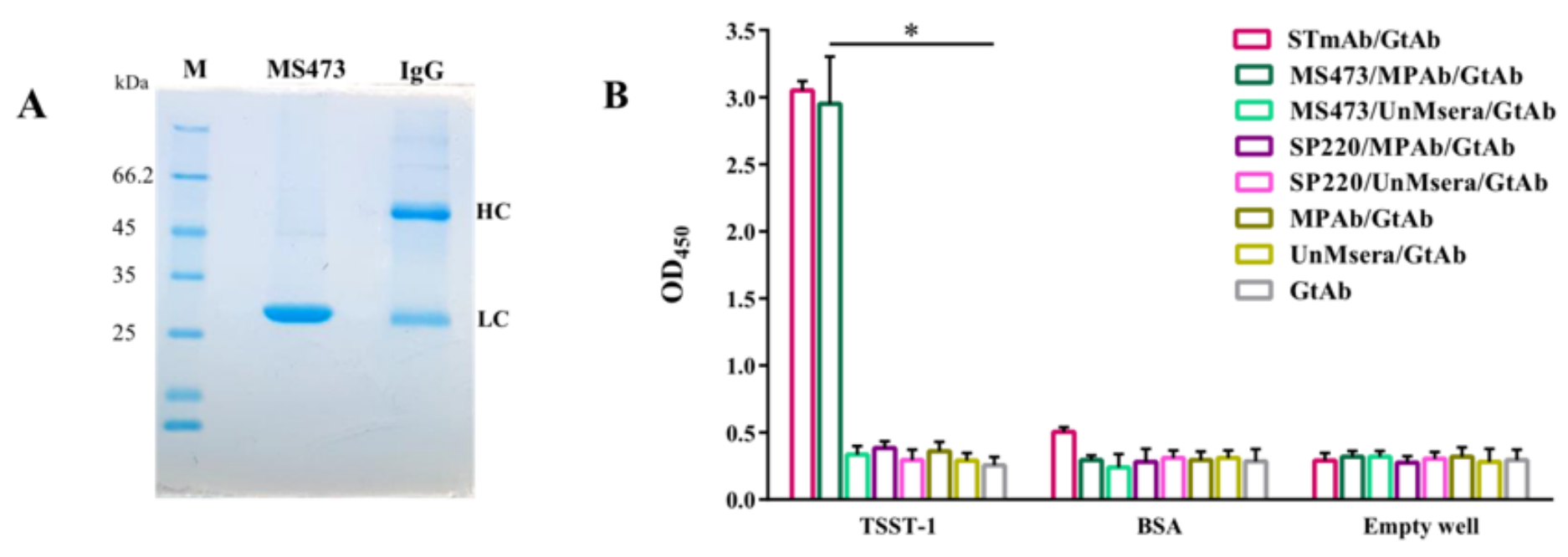

\section{Figure 3}

The significant binding of MS473 to TSST-1. The purity and TSST-1 binding ability of MS473 was evaluated by (A) SDS-PAGE and (B) ELISA. (A) A single protein band at approximately $27 \mathrm{kDa}$ related to the purified ScFv is seen on a $12 \%$ SDS-PAGE gel stained with Coomassie ${ }^{\circledR}$ blue. IgG: human immunoglobulin G, Lane M: unstained protein marker. The original image can be found in Supplementary Fig. S3. (B) The wells coated with TSST-1 or bovine serum albumin (BSA) (control), and empty wells (control) were individually incubated with MS473 or SP220, followed by mouse anti-human scFv polyclonal antibody (MPAb) or unimmunized mouse sera (UnMsera; control). Next, the wells were incubated with goat anti-mouse IgG- horseradish peroxidase (HRP)-conjugated antibody (GtAb), followed by the addition of the TMB solution. Other control groups include the TSST-1-coated wells, BSA-coated wells, and empty wells incubated directly with anti-staphylococcal TSST-1 mAb (STmAb) followed by GtAb; incubated directly with MPAb, followed by GtAb; incubated directly with UnMsera, followed by GtAb; or incubated directly with GtAb. Samples were run in triplicate, and the results are expressed as mean \pm SEM of at least three individual experiments. Statistical comparisons were carried out with the one-way analysis of variance (ANOVA). ${ }^{*} P<0.05$. 


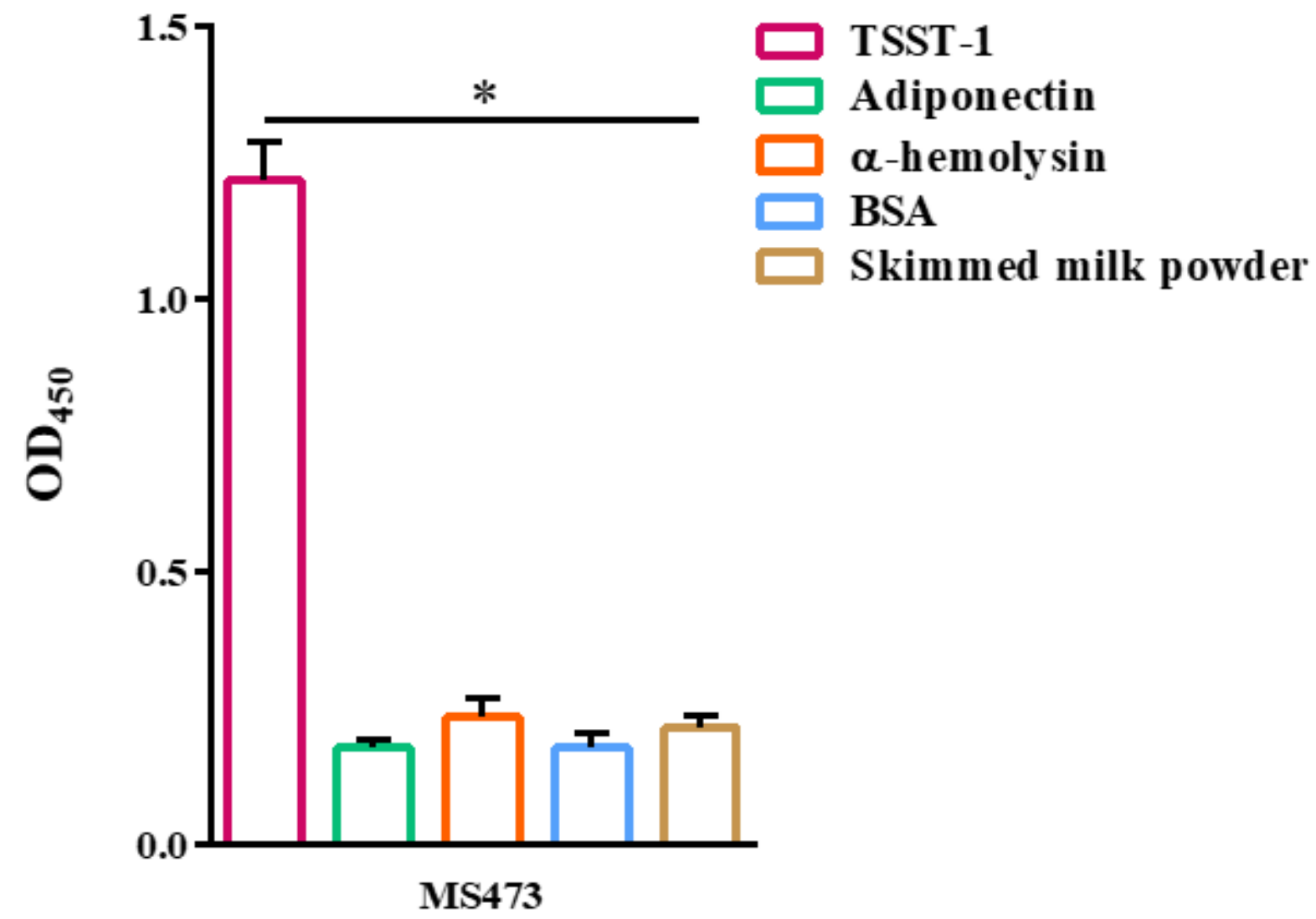

Figure 4

TSST-1-specific binding of MS473. The specific binding of MS473 to TSST-1 was evaluated by ELISA. The wells coated with adiponectin, a-hemolysin, bovine serum albumin (BSA), skimmed milk powder, and TSST-1 were incubated with MS473, followed by mouse anti-human ScFv polyclonal antibody and goat anti-mouse lgG-horseradish peroxidase (HRP)-conjugated antibody. The MS473 scFv exhibited significant binding to TSST-1 compared to the control proteins. Samples were run in triplicate, and the results are expressed as mean \pm SEM of at least three individual experiments. Statistical comparisons were carried out with the one-way analysis of variance (ANOVA). ${ }^{\star} P<0.05$. 


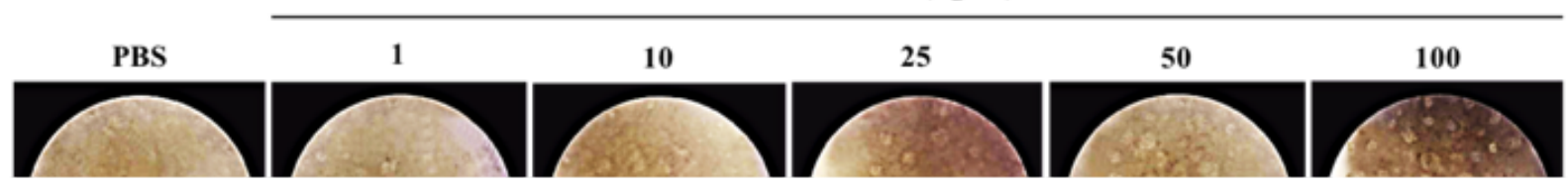

\section{Figure 5}

Inhibition of the mitogenic response of human peripheral blood mononuclear cells (PBMCs) to TSST-1 by MS473. (A) To determine the effective proliferative dose of TSST-1, fresh human PBMCs $\left(\sim 10^{6} \mathrm{cells} / \mathrm{ml}\right)$ were incubated with the TSST-1 protein at concentrations of 1,10,25, 50, and $100 \mathrm{ng} / \mathrm{ml}$ for 24 hours at $37^{\circ} \mathrm{C}, 5 \% \mathrm{CO}$. The cells incubated with PBS served as the control. The proliferation of PBMCs and the formation of cell clumps induced by TSST-1 were investigated using an inverted microscope. (B) Fresh human PBMCs $\left(\sim 10^{6}\right.$ cells $\left./ \mathrm{ml}\right)$ were incubated simultaneously with the TSST-1 protein $(50 \mathrm{ng} / \mathrm{ml})$ and MS473 $(80 \mu \mathrm{g} / \mathrm{ml})$ for 24 hours at $37^{\circ} \mathrm{C}, 5 \%$ CO2. The cells incubated with PBS or the TSST-1 $(50 \mathrm{ng} / \mathrm{ml})$ and PBS served as the controls. The MS473 scFv exhibited a significant inhibitory effect on PBMCs proliferation and decreased the number of colony-forming cells. 


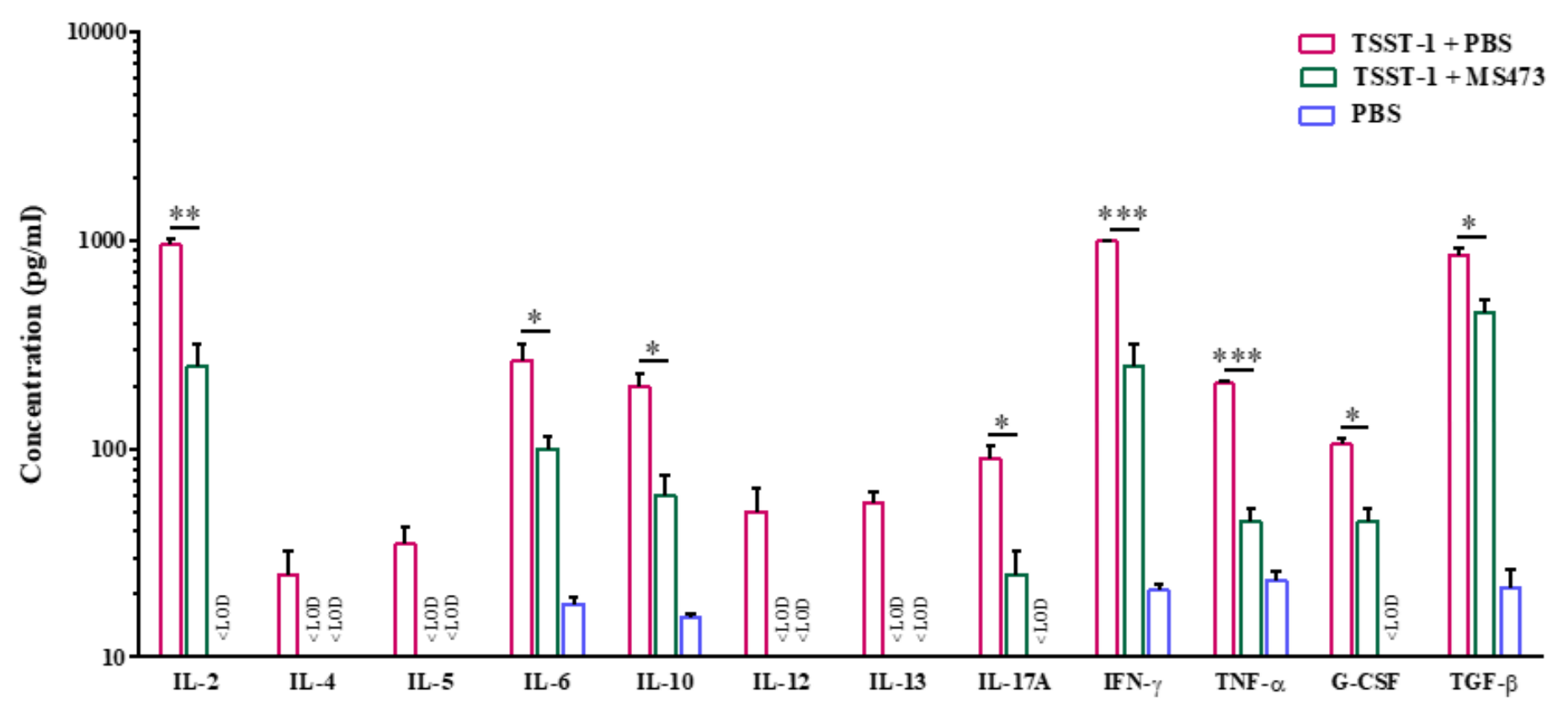

Figure 6

Inhibition Effects of MS473 on the production of cytokines from human peripheral blood mononuclear cells (PBMCs) stimulated with TSST-1. To assess the inhibition effect of MS473 on the TSST-1-induced cytokine secretion, fresh human PBMCs $\left(\sim 10^{6} \mathrm{cell} / \mathrm{ml}\right)$ were incubated simultaneously with the TSST-1 protein $(50 \mathrm{ng} / \mathrm{ml})$ and MS473 $(80 \mu \mathrm{g} / \mathrm{ml})$ for 24 hours at $37^{\circ} \mathrm{C}, 5 \% \mathrm{CO} 2$. The cells treated with PBS or the TSST-1 protein $(50 \mathrm{ng} / \mathrm{ml})$ and PBS served as the controls. The concentration of human interleukin 2 (IL)-2, IL-4, IL-5, IL-6, IL-10, IL-12, IL-13, IL-17A, interferon (IFN)-y, tumor necrosis factor (TNF)-a, G-CSF, and transforming growth factor (TGF) $\beta$ in the culture supernatant of PBMCs (stimulated and unstimulated) were calculated using the standard curve provided in a Multi-Analyte ELISArray Kit (Qiagen). The concentration of cytokines $(\mathrm{pg} / \mathrm{ml})$ is represented on a logarithmic scale. The concentrations of IL-4, IL-5, IL-12, and IL-13 in the culture supernatant of PBMCs treated with TSST1 and MS473 (or PBS) were less than the limit of detection (LOD). Moreover, the concentrations of IL-2, IL-17A, and G-CSF in the culture supernatant of PBMCs treated with PBS were less than the LOD. Samples were run in triplicate, and the results are expressed as mean \pm SD. ${ }^{\star} P<0.05, * \star P=0.01$, $* \star \star P<0.01$.

\section{Supplementary Files}

This is a list of supplementary files associated with this preprint. Click to download.

- supplementaryfile2.pdf 